Annales Mathematicae Silesianae 30 (2016), 129-142

DOI: 10.1515/amsil-2016-0002

\title{
STRONG UNIQUE ERGODICITY OF RANDOM DYNAMICAL SYSTEMS ON POLISH SPACES
}

\author{
PAWeŁ PŁonka
}

\begin{abstract}
In this paper we want to show the existence of a form of asymptotic stability of random dynamical systems in the sense of L. Arnold using arguments analogous to those presented by T. Szarek in [6], that is showing it using conditions generalizing the notion of tightness of measures. In order to do that we use tightness theory for random measures as developed by $\mathrm{H}$. Crauel in 2 .
\end{abstract}

\section{Introduction}

In the theory of random dynamical systems it is still usual to show asymptotic stability or the existence of random attractors by showing the existence of invariant compact random set. This may be problematic especially if the phase space is infinite dimensional. This paper shows weaker criteria for a form of asymptotic stability of random dynamical systems, that is for the existence of a unique invariant random probability measure such that any random probability measure will evolve under the action of the random dynamical system towards it.

In order to transfer this results to the case of random dynamical systems in the sense of L. Arnold (see [1]) we will use the Prokhorov theory developed for random probability measures on Polish spaces by $\mathrm{H}$. Crauel in [2] following M. Valadier [7, which was used in the theory of random attractors and

Received: 22.05.2015. Revised: 29.02.2016. Accepted:

(2010) Mathematics Subject Classification: 37H99, 37B25, 47B80, 60H25.

Key words and phrases: random dynamical systems, invariant measures, asymptotic stability. 
associated invariant measures developed by H. Crauel and F. Flandoli in [3]. We will use this theory to introduce criteria for the existence of invariant measures as well as asymptotic stability of measures associated with random dynamical spaces on Polish spaces.

Let assume that $\varphi$ is a continuous random dynamical system over Polish space $X$ and measurable dynamical system $\left(\Omega, \mathcal{F}, \mathbf{P}, \theta_{t \in \mathbb{T}}\right)$ where $\mathbb{T} \in$ $\left\{\mathbb{Z}^{+}, \mathbb{R}^{+}\right\}$, and $\Phi(t)$ is an associated skew product as defined in section 2 . Associated with $\varphi$ is a space of probability measures over $\mathcal{F} \otimes \mathcal{B}(X)$ also defined below and denoted $\mathcal{P}_{\Omega}(X)$. Here we will assume that $\mathcal{P}_{\Omega}(X)$ is a Polish space with metric $d$ which happens if and only if $\Omega$ is countably generated (see [2]). Under such assumption we can define a class of non-expansive random dynamical systems such that $d(\Phi(t) \mu, \Phi(t) \nu) \leq d(\mu, \nu)$ for all $t \in \mathbb{T}$ and $\mu, \nu \in \mathcal{P}_{\Omega}(X)$. We are interested in finding a $\varphi$-invariant measure $\mu_{0} \in \mathcal{P}_{\Omega}(X)$ such that $\Phi(t) \mu \rightarrow \mu_{0}$ in the topology of weak convergence for all $\mu \in \mathcal{P}_{\Omega}(X)$, for nonexpansive random dynamical systems. Note that because of non-expansiveness (and using the fact that $\Phi(t+s)=\Phi(t) \Phi(s)$ ) it is enough to show this for some discrete random dynamical system defined by $\left\{\Phi\left(n t_{0}\right)\right\}_{n \in \mathbb{N}}$ where $t_{0}>0$, that is why we treat only discrete case for the rest of the paper.

\section{Basic definitions and notations}

Let $X$ be a Polish space, that is a separable completely metrizable topological space. We will always assume that $X$ is equipped with a complete metric $d(x, y)$. By $B(x, \varepsilon)$ we will denote a closed ball centered at $x$ with radius $\varepsilon$. If $A \subseteq X$, then by $A^{\varepsilon, 0}$ we will denote an $\varepsilon$-neighborhood of $A$, that is

$$
A^{\varepsilon, 0}:=\{x \in X: d(x, A)<\varepsilon\},
$$

where $d(x, A):=\inf \{d(x, y): y \in A\}$. By $A^{\varepsilon}$ we will denote a closed $\varepsilon$ neighborhood of $A$, that is

$$
A^{\varepsilon}:=\{x \in X: d(x, A) \leq \varepsilon\} .
$$

By $\mathcal{B}(X)$ we will denote Borel subsets of $X$, and by $\mathcal{B}_{b}(X)$ we will denote bounded Borel subsets of $X$. If $A \in \mathcal{B}(X)$, then diam $A:=\sup \{d(x, y): x, y \in$ $A$ \} denotes the diameter of $A$. By $\mathcal{C}_{\varepsilon}(X)$ we will denote a family of $\varepsilon$-compact subsets of $X$, that is if $A \in \mathcal{C}_{\varepsilon}(X)$ then $A$ is closed and there exists $n \in \mathbb{N}$ and 
$x_{1}, \ldots, x_{n} \in X$ such that

$$
A \subseteq \bigcup_{i=1}^{n} B\left(x_{i}, \varepsilon\right)
$$

Furthermore by $\mathcal{C}_{\varepsilon}^{k}(X)$ we will denote those sets $A \in \mathcal{C}_{\varepsilon}(X)$ for which there are $x_{1}, \ldots, x_{k} \in X$ such that $A \subseteq \bigcup_{i=1}^{k} B\left(x_{i}, \varepsilon\right)$.

Let us denote by $(\Omega, \mathcal{F}, \mathbf{P})$ a probability space and by $\mathbb{T} \in\left\{\mathbb{Z}, \mathbb{Z}^{+}, \mathbb{R}, \mathbb{R}^{+}\right\}$ the time space. By $\mathcal{P}_{\Omega}(X)$ we will denote the set of probability measures on $(\Omega \times X, \mathcal{F} \otimes \mathcal{B}(X))$, such that if $\mu \in \mathcal{P}_{\Omega}(X)$, then $\pi_{\Omega} \mu(B)=\mu\left(\pi_{\Omega}^{-1}(B)\right)=$ $\mathbf{P}(B)$ for all $B \in \mathcal{B}(X)$, where $\pi_{\Omega}(\omega, x)=\omega$ is a projection on $\Omega$. As stated in [1] such measures correspond to functions $\mu .(\cdot): \Omega \times \mathcal{B}(X) \rightarrow[0,1]$ such that $\omega \mapsto \mu_{\omega}(B)$ is a measurable function for every $B \in \mathcal{B}(X)$, the function $B \mapsto \mu_{\omega}(B)$ is a probability measure for each $\omega \in \Omega$ and such that

$$
\mu(A)=\int_{\Omega} \int_{X} \mathbf{1}_{A}(\omega, x) \mu_{\omega}(d x) \mathbf{P}(d \omega)
$$

for $A \in \mathcal{F} \otimes \mathcal{B}(X)$, is a measure in $\mathcal{P}_{\Omega}(X)$. If $\mu \in \mathcal{P}_{\Omega}(X)$ then the corresponding $\mu_{\omega}$ is called a factorization of $\mu$ with respect to $\mathbf{P}$. By $\mathcal{P}_{\Omega}^{A}(X)$ we will denote the set of measures in $\mathcal{P}_{\Omega}(X)$ which are supported on $A \in \mathcal{F} \otimes \mathcal{B}(X)$, that is if $\mu \in \mathcal{P}_{\Omega}^{A}(X)$ then $\mu(A)=1$. By $\mathcal{P}(X)$ we will denote probability measures on $(X, \mathcal{B}(X))$. Note that if $\mu \in \mathcal{P}(X)$ then $\omega \mapsto \mu$ describes the corresponding measure in $\mathcal{P}_{\Omega}(X)$.

Let us denote by $\mathcal{C}_{b}(X)$ the set of all continuous and bounded real functions. The space $\mathcal{C}_{b}(X)$ is equipped with the standard supremum norm. Following [1] we will introduce the space $\mathcal{L}_{\mathbf{P}}^{1}\left(\Omega, \mathcal{C}_{b}(X)\right)$ of functions $f: \Omega \rightarrow \mathcal{C}_{b}(X)$ measurable and such that $\|f\|:=\int_{\Omega}\|f(\omega, \cdot)\| d \mathbf{P}<\infty$. Note that if $f \in \mathcal{C}_{b}(X)$ then $(\omega, x) \mapsto f(x)$ describes the corresponding function in $\mathcal{L}_{\mathbf{P}}^{1}\left(\Omega, \mathcal{C}_{b}(X)\right)$. For any measure $\mu$ and function $f$ let us introduce notation $\mu(f)=\int f d \mu$. The smallest topology on $\mathcal{P}_{\Omega}(X)$ which makes the operator $f \mapsto \mu(f)$ continuous, where $f \in \mathcal{L}_{\mathbf{P}}^{1}\left(\Omega, \mathcal{C}_{b}(X)\right)$ is called the topology of weak convergence on $\mathcal{P}_{\Omega}(X)$. Similarly the topology of weak convergence for $\mathcal{P}(X)$ is understood by us as the smallest topology which makes $f \mapsto \mu(f)$ continuous for $f \in \mathcal{C}_{b}(X)$ and $\mu \in \mathcal{P}(X)$.

By $\left(\theta_{t}\right)_{t \in \mathbb{T}}$ we will denote a metric dynamical system over $\Omega$. That is it satisfies the following conditions

$$
(\omega, t) \longmapsto \theta_{t}(\omega)
$$

is measurable,

$$
\theta_{0}(\omega)=i d(\omega)
$$




$$
\theta_{t+s}(\omega)=\theta_{t} \circ \theta_{s}(\omega)
$$

for $s, t \in \mathbb{T}$,

$$
\theta_{t} \mathbf{P}(F)=\mathbf{P}\left(\theta_{t}^{-1} F\right)=\mathbf{P}(F)
$$

for $t \in \mathbb{T}, F \in \mathcal{F}$.

Let $\left(\theta_{t}\right)_{t \in \mathbb{T}}$ be a metric dynamical system. We call a function $\varphi: \mathbb{T} \times \Omega \times$ $X \rightarrow X$ a random dynamical system if it is $\mathcal{B}(\mathbb{T}) \otimes \mathcal{F} \otimes \mathcal{B}(X)$ measurable, the function $\varphi(t, \omega, \cdot): X \rightarrow X$ is continuous and $\varphi$ satisfies the following cocycle property

$$
\varphi(0, \omega)=i d_{X}
$$

for $\omega \in \Omega$,

$$
\varphi(t+s, \omega)=\varphi\left(t, \theta_{s}(\omega)\right) \circ \varphi(s, \omega)
$$

for $s, t \in \mathbb{T}, \omega \in \Omega$. The following family of mappings is called a skew product on $\varphi$

$$
(\omega, x) \mapsto\left(\theta_{t} \omega, \varphi(t, \omega) x\right)=: \Phi(t)(\omega, x)
$$

for $t \in \mathbb{T}$. Skew product is a measurable dynamical system on $(\Omega \times X, \mathcal{F} \otimes$ $\mathcal{B}(X))$. With the following notations $\Phi(t) \mu(\cdot)=\mu\left(\Phi(t)^{-1}(\cdot)\right)$ and $\Phi(t) f(\omega, x)=$ $f(\Phi(t)(\omega, x))$ we have $\Phi(t) \mu(f)=\mu(\Phi(t) f), \Phi(t)$ is a continuous linear mapping on $\mathcal{L}_{\mathbf{P}}^{1}\left(\Omega, \mathcal{C}_{b}(X)\right)$ and a continuous affine mapping on $\mathcal{P}_{\Omega}(X)$.

Given a random dynamical system $\varphi$ we will call a measure $\mu \in \mathcal{P}_{\Omega}(X)$ $\varphi$-invariant if it satisfies

$$
\Phi(t) \mu=\mu
$$

for $t \in \mathbb{T}$. We denote the set of all $\varphi$-invariant measures by $\mathcal{I}_{\mathbf{P}}(\varphi)$.

We call a random dynamical system to have the property of $\varphi$ strong unique ergodicity if there exists $\nu \in \mathcal{I}_{\mathbf{P}}(\varphi)$ such that

$$
\lim _{t \rightarrow \infty} \Phi(t) \mu=\nu
$$

for all $\mu \in \mathcal{P}_{\Omega}(X)$. Notice that in this case the measure $\nu$ is unique.

In the rest of the paper we will mainly deal with discrete dynamical systems, so unless stated otherwise assume that $\mathbb{T}=\mathbb{Z}^{+}$. 


\subsection{Basic facts}

Let us denote by $\pi_{X}(\omega, x):=x$ the projection of $\Omega \times X$ onto $X$. For every $\mu \in \mathcal{P}_{\Omega}(X)$ we use the following notation $\pi_{X} \mu(A)=\mu\left(\pi_{X}^{-1}(A)\right)=\mathbb{E} \mu \cdot(A)$ for $A \in \mathcal{B}(X)$. Following H. Crauel (see [2, Chapter 4]) we introduce notion of tightness of measures.

Definition 2.1. We say that a set $\Gamma \subseteq \mathcal{P}(X)$ of Borel probability measures on $X$ is tight if for every $\varepsilon>0$ there exists a compact set $K \subseteq X$ such that

$$
\mu(K) \geq 1-\varepsilon
$$

for every $\mu \in \Gamma$. We say that a set $\Gamma \subseteq \mathcal{P}_{\Omega}(X)$ of probabilities on $X \times \Omega$ is tight if the set $\pi_{X} \Gamma=\left\{\pi_{X} \mu: \mu \in \Gamma\right\}$ is tight.

H. Crauel showed that $\pi_{X}: \mathcal{P}_{\Omega}(X) \rightarrow \mathcal{P}(X)$ is a continuous surjective mapping such that if $K \subseteq \mathcal{P}(X)$ is compact, then $\pi_{X}^{-1}(K)$ is compact in $\mathcal{P}_{\Omega}(X)$ (see [2, Theorem 4.14]). From this he obtains the following Prokhorovtype theorem.

TheOrem 2.2. Suppose that $\Gamma \subseteq \mathcal{P}_{\Omega}(X)$. Then $\Gamma$ is tight if and only if it is relatively compact with respect to the topology of weak convergence. In this case it is also relatively sequentially compact.

Let $\varphi$ be a discrete random dynamical system and $\Phi(n)$ its skew product associated with $\varphi$. Assume that there exists $\mu \in \mathcal{P}_{\Omega}(X)$ such that the family $\{\Phi(n) \mu\}_{n \in \mathbb{N}}$ is tight. Then the sequence of measures $\left\{\nu_{n}\right\}_{n \in \mathbb{N}}$ defined by

$$
\nu_{n}(\cdot):=\frac{1}{n} \sum_{k=1}^{n} \Phi(k) \mu(\cdot)
$$

for $n \in \mathbb{N}$, is also tight. Then Theorem 2.2 says that it has some convergent subsequence with a limit $\nu_{*}$. From [1, Theorem 1.5.8] we know that $\nu_{*} \in$ $\mathcal{I}_{\mathbf{P}}(\varphi)$, that is $\nu_{*}$ is a $\varphi$-invariant measure.

Now we will prove a lemma which we will use later on.

Lemma 2.3. Let $\mu \in \mathcal{P}_{\Omega}(X)$. If for every $\varepsilon>0$ there exists a set $C_{\varepsilon} \in$ $\mathcal{C}_{\varepsilon}(X)$ such that

$$
\pi_{X} \Phi(n) \mu\left(C_{\varepsilon}\right) \geq 1-\varepsilon
$$

for $n \in \mathbb{N}$, then $\{\Phi(n) \mu\}_{n \in \mathbb{N}}$ is tight. 
Proof. Let $\varepsilon>0$. For each $\frac{\varepsilon}{2^{n}}$ chose $C_{\frac{\varepsilon}{2^{n}}}$ by assumption. Set $K:=$ $\bigcap_{k=1}^{\infty} C_{\frac{\varepsilon}{2^{k}}}$. Since $X$ is a Polish space, $K$ is a compact set and we have

$$
\begin{aligned}
\pi_{X} \Phi(n) \mu(X \backslash K) & =\pi_{X} \Phi(n) \mu\left(\bigcup_{k=1}^{\infty} X \backslash C_{\frac{\varepsilon}{2^{k}}}\right) \\
& \leq \sum_{k=1}^{\infty} \pi_{X} \Phi(n) \mu\left(X \backslash C_{\frac{\varepsilon}{2^{k}}}\right) \leq \sum_{k=1}^{\infty} \frac{\varepsilon}{2^{k}}=\varepsilon .
\end{aligned}
$$

Which in accordance with Definition 2.1 shows that $\{\Phi(n) \mu\}_{n \in \mathbb{N}}$ is tight.

In his book $\mathrm{H}$. Crauel also showed that $\mathcal{P}_{\Omega}(X)$ is a Polish space if and only if the space $(\Omega, \mathcal{F}, \mathbf{P})$ is countably generated, that is there exists a set of generators $\mathcal{F}, \subseteq \mathcal{F}$ which is countable and for every $F \in \mathcal{F}$ there exists $G \in \mathcal{F}$, such that $\mathbf{P}(F \Delta G)=0$. We will maintain this assumption through the rest of the paper. In that case we can define the following metric.

Definition 2.4. Let $\mathcal{F}_{1}=\left\{G_{m}: m \in \mathbb{N}\right\}$ be a set of generators for $\mathcal{F}$, such that $G_{0}=\Omega$. For $\mu, \nu \in \mathcal{P}_{\Omega}(X)$ we define

$$
\begin{array}{r}
d(\mu, \nu):=\sum_{m=0}^{\infty} \frac{1}{2^{m}} \sup \left\{\int_{G_{m}}\left|\mu_{\omega}(f)-\nu_{\omega}(f)\right| \mathbf{P}(d \omega): f \in \mathcal{C}_{b}(X), 0 \leq f \leq 1,\right. \\
|f(x)-f(y)| \leq d(x, y), x, y \in X\} .
\end{array}
$$

Notice that the metric $d$ depends on the choice of the generating family $\mathcal{F}_{1}$, but of course all are equivalent in the sense that they generate the same topology. In this paper we need it only to show convergence in the weak topology, and the dependence on the generating family may be relevant only in the definition of non-expansiveness of $\varphi$ where it will be noticed. Now we will prove two lemmas that we will use later on. Notice that they are not dependent on the choice of the generating family.

Lemma 2.5. Let $\varepsilon>0$ and $\mu_{1}, \mu_{2} \in \mathcal{P}_{\Omega}(X)$. If $d\left(\mu_{1}, \mu_{2}\right) \leq \varepsilon^{2}$ then

$$
\pi_{X} \mu_{1}\left(A^{\varepsilon}\right) \geq \pi_{X} \mu_{2}(A)-\varepsilon
$$

for $A \in \mathcal{B}(X)$.

Proof. Take $A \in \mathcal{B}(X)$ and define $f(x):=\max \{\varepsilon-d(x, A), 0\}$. Then we have

$$
\varepsilon \mu_{i, \omega}(A) \leq \mu_{i, \omega}(f) \leq \varepsilon \mu_{i, \omega}\left(A^{\varepsilon}\right)
$$


for $i=1,2$ and $\omega \in \Omega$, where $\mu_{i, \omega}$ denotes the disintegration of $\mu_{i}$. From this we obtain

$$
\begin{aligned}
\varepsilon \pi_{X} \mu_{2}(A)- & \varepsilon \pi_{X} \mu_{1}\left(A^{\varepsilon}\right)=\varepsilon \int_{\Omega} \mu_{2, \omega}(A) \mathbf{P}(d \omega)-\varepsilon \int_{\Omega} \mu_{1, \omega}\left(A^{\varepsilon}\right) \mathbf{P}(d \omega) \\
\leq & \int_{\Omega}\left(\mu_{2, \omega}(f)-\mu_{1, \omega}(f)\right) \mathbf{P}(d \omega) \\
\leq & \sup \left\{\int_{\Omega=G_{0}}\left|\mu_{2, \omega}(f)-\mu_{1, \omega}(f)\right| \mathbf{P}(d \omega): f \in \mathcal{C}_{b}(X), 0 \leq f \leq 1,\right. \\
& |f(x)-f(y)| \leq d(x, y), x, y \in X\} \\
\leq & \sum_{m=1}^{\infty} \frac{1}{2^{m}} \sup \left\{\int_{G_{m}}\left|\mu_{2, \omega}(f)-\mu_{1, \omega}(f)\right| \mathbf{P}(d \omega): f \in \mathcal{C}_{b}(X),\right. \\
& 0 \leq f \leq 1,|f(x)-f(y)| \leq d(x, y), x, y \in X\} \\
= & d\left(\mu_{1}, \mu_{2}\right) \leq \varepsilon^{2} .
\end{aligned}
$$

Which after rearrangement gives us

$$
\pi_{X} \mu_{1}\left(A^{\varepsilon}\right) \geq \pi_{X} \mu_{2}(A)-\varepsilon .
$$

For small $\varepsilon>0$ that is true even if $\Omega$ has a different position in generating family.

Lemma 2.6. Let $A \in \mathcal{B}_{b}(X)$ be nonempty and $\mu, \nu \in \mathcal{P}_{\Omega}^{\pi_{X}^{-1} A}(X)$. Then

$$
d(\mu, \nu) \leq 4 \operatorname{diam} A .
$$

Proof. Let $y \in A$ and let $f \in \mathcal{C}_{b}(X)$ be such that $0 \leq f \leq 1$ and $|f(x)-f(y)| \leq d(x, y)$ for $x, y \in X$. Then

$$
\begin{aligned}
\left|\mu_{\omega}(f)-\nu_{\omega}(f)\right| & \leq\left|\mu_{\omega}(f)-f(y)\right|+\left|f(y)-\nu_{\omega}(f)\right| \\
& =\left|\int_{A}(f(x)-f(y)) \mu_{\omega}(d x)\right|+\left|\int_{A}(f(y)-f(x)) \nu_{\omega}(d x)\right| \\
& \leq \int_{A} d(x, y) \mu_{\omega}(d x)+\int_{A} d(x, y) \nu_{\omega}(d x) \\
& \leq \operatorname{diam} A+\operatorname{diam} A=2 \operatorname{diam} A
\end{aligned}
$$


which is true for $\mathbf{P}$-almost all $\omega \in \Omega$. From this and Definition 2.4 we obtain

$$
\begin{aligned}
d(\mu, \nu) & \leq 2 \operatorname{diam} A \sum_{m \in \mathbb{N}} \frac{1}{2^{m}} \mathbf{P}\left(G_{m}\right) \\
& \leq 2 \sum_{m \in \mathbb{N}} \frac{1}{2^{m}} \operatorname{diam} A=4 \operatorname{diam} A .
\end{aligned}
$$

\section{Criteria for the existence of an invariant measure}

Now we will introduce the definition of the concentration of random dynamical systems. Following the definition there is an example of a random dynamical system which is concentrating and which doesn't have an invariant measure.

Definition 3.1. We say that $\varphi$ is concentrating if for every $\varepsilon>0$ there exists $A \in \mathcal{B}(X)$ with $\operatorname{diam} A \leq \varepsilon$ and $\alpha>0$ such that

$$
\liminf _{n \rightarrow \infty} \pi_{X} \Phi(n) \mu(A) \geq \alpha
$$

for all $\mu \in \mathcal{P}_{\Omega}(X)$.

ExAmPLE 3.2. Let $\mathbb{H}$ be an infinite dimensional separable Hilbert space and let us denote the orthonormal basis of it using the following subset $\mathbb{B}=$ $\left\{u_{1}, u_{2}, \ldots\right\} \cup\left\{v_{1}, v_{2}, \ldots\right\} \subseteq \mathbb{H}$. Let us also choose real numbers $r_{1}, r_{2}, \ldots \in \mathbb{R}$ such that $\frac{\sqrt{2}}{2}>r_{1}>r_{2}>\ldots$ and $\lim _{n \rightarrow \infty} r_{n}=0$ and for each $i \in \mathbb{N}$ define $X_{i}=u_{i}+r_{i} S(0,1)$ where $S(0,1)=\left\{\sum_{k=1}^{\infty} \alpha_{k} v_{k} \in \mathbb{H}: \sum_{k=1}^{\infty} \alpha_{k}^{2}=1\right\}$. Let $X=\bigcup_{i=1}^{\infty} X_{i}$. Observe that $X_{i}$ are closed subsets of $\mathbb{H}$ and $X_{i} \cap X_{j}=\emptyset$ for $i \neq j$ which makes $X$ a closed subset of $\mathbb{H}$ and thus a Polish space. Now for each $m \in \mathbb{N}$ define $T_{m}: X \rightarrow X_{m}$ such that for each $x=u_{i}+r_{i} \sum_{k=1}^{\infty} \alpha_{k} v_{k} \in X$ we have

$$
T_{m}\left(u_{i}+r_{i} \sum_{k=1}^{\infty} \alpha_{k} v_{k}\right)=u_{m}+r_{m} \sum_{k=1}^{\infty} \alpha_{k} v_{k+1} .
$$

Now let us define $\Omega=\mathbb{N}^{\mathbb{Z}^{+}}$, let $\mathcal{F}$ be the product $\sigma$-algebra and let $\mathbf{P}$ be defined by

$$
\mathbf{P}\left(\left\{\omega \in \Omega: \omega_{0}=i_{0}, \ldots, \omega_{k}=i_{k}\right\}\right)=\frac{1}{2^{i_{0}}} \cdot \ldots \cdot \frac{1}{2^{i_{k}}}
$$


and canonically extended to the whole of $\mathcal{F}$. Now let us define a random dynamical system $\varphi$ by the formula

$$
\varphi(n, \omega)= \begin{cases}T_{\left(\theta_{n-1} \omega\right)_{0}} \circ \ldots \circ T_{\omega_{0}} & \text { for } n \geq 1 \\ i d_{X} & \text { for } n=0\end{cases}
$$

Then one can see that $\pi_{X} \Phi(n) \mu\left(X_{l}\right)=\frac{1}{2^{l}}$ for $\mu \in \mathcal{P}_{\Omega}(X)$ and $l \in \mathbb{N}$. Since $\operatorname{diam} X_{i}=r_{i}$ for each $i \in \mathbb{N}$ the condition of concentration holds trivially as for every $\varepsilon>0$ we can choose $r_{l}<\varepsilon$ and fix $A=X_{l}$ and $\alpha=\frac{1}{2^{l}}$. Suppose that there exists some $\varphi$-invariant measure $\mu_{0} \in \mathcal{P}_{\Omega}(X)$. It is possible to show that if $x \in \operatorname{supp} \pi_{X} \Phi(n) \mu_{0}$ and $x=u_{i}+r_{i} \sum_{k=1}^{\infty} \alpha_{k} v_{k}$ then $\alpha_{n}=0$. Since $\operatorname{supp} \pi_{X} \Phi(n) \mu_{0}=\operatorname{supp} \pi_{X} \mu_{0}$ for every $n \in \mathbb{N}$ we obtain that $\operatorname{supp} \pi_{X} \mu_{0}=\emptyset$.

Below we introduce the definition of non-expansiveness. Notice that this condition may be dependent on the choice of generating family in the definition of metric $d$. For this paper its enough that this condition will hold for just one such choice.

DEFINITION 3.3. We say that $\varphi$ is non-expansive if

$$
d(\Phi(1) \mu, \Phi(1) \nu) \leq d(\mu, \nu)
$$

for all $\mu, \nu \in \mathcal{P}_{\Omega}(X)$.

Now we prove the main theorem in the form of two lemmas followed by main assertion of this paper.

LEMMA 3.4. If $\varphi$ is non-expansive and concentrating then there exists a $\varphi$-invariant measure $\nu \in \mathcal{I}_{\mathbf{P}}(\varphi)$.

Proof. Let us take arbitrary $\varepsilon>0$ and $\mu \in \mathcal{P}_{\Omega}(X)$. Put $\bar{\varepsilon}=\frac{\varepsilon^{2}}{64}$ and choose $A \in \mathcal{B}(X)$ and $\alpha>0$ from the definition of concentration for $\bar{\varepsilon}$. Let us define a new measure $\nu_{n}$ for each $n \in \mathbb{N}$ by

$$
\nu_{n}(B):=\frac{\Phi(n) \mu\left(B \cap \pi_{X}^{-1}(A)\right)}{\Phi(n) \mu\left(\pi_{X}^{-1}(A)\right)}
$$

for $B \in \mathcal{F} \times \mathcal{B}(X)$. Observe that

$$
\begin{aligned}
\pi_{X} \nu_{n}(B) & =\nu_{n}\left(\pi_{X}^{-1}(B)\right)=\frac{\Phi(n) \mu\left(\pi_{X}^{-1}(B) \cap \pi_{X}^{-1}(A)\right)}{\Phi(n) \mu\left(\pi_{X}^{-1}(A)\right)} \\
& =\frac{\Phi(n) \mu\left(\pi_{X}^{-1}(B \cap A)\right)}{\Phi(n) \mu\left(\pi_{X}^{-1}(A)\right)}=\frac{\pi_{X} \Phi(n) \mu(B \cap A)}{\pi_{X} \Phi(n) \mu(A)}
\end{aligned}
$$


for $B \in \mathcal{B}(X)$. Since $\varphi$ is concentrating, we can define

$$
\delta:=\sup \left\{\gamma>0: \exists_{C_{\frac{\varepsilon}{2}} \in \mathcal{C}_{\frac{\varepsilon}{2}}(X)} \liminf _{n \rightarrow \infty} \pi_{X} \Phi(n) \mu\left(C_{\frac{\varepsilon}{2}}\right) \geq \gamma\right\} .
$$

Let $C_{\frac{\varepsilon}{2}} \in \mathcal{C}_{\frac{\varepsilon}{2}}(X)$ be chosen in accordance to the definition of $\delta$. Then we have

$$
\pi_{X} \Phi(n) \nu\left(C_{\frac{\varepsilon}{2}}^{\frac{\varepsilon}{2}}\right) \geq 1-\frac{\varepsilon}{2}
$$

for all $n \in \mathbb{N}$ and $\nu \in \mathcal{P}_{\Omega}(X)$ which are supported on $\pi^{-1}(A)$. We show this by contradiction. Suppose that there exists some $n_{0} \in \mathbb{N}$ and $\nu_{0} \in \mathcal{P}_{\Omega}(X)$ supported on $\pi_{X}^{-1}(A)$ such that

$$
\pi_{X} \Phi\left(n_{0}\right) \nu_{0}\left(C_{\frac{\varepsilon}{2}}^{\frac{\varepsilon}{2}}\right)<1-\frac{\varepsilon}{2} .
$$

By Ulam's theorem there exists a compact set $K \subseteq X \backslash C_{\frac{\varepsilon}{2}}^{\frac{\varepsilon}{2}}$ such that

$$
\pi_{X} \Phi\left(n_{0}\right) \nu_{0}(K) \geq \frac{\varepsilon}{2}
$$

By non-expansiveness of $\varphi$ we have

$$
d\left(\Phi\left(n_{0}\right) \nu_{0}, \Phi\left(n_{0}\right) \nu\right) \leq d\left(\nu_{0}, \nu\right)
$$

for any $\nu \in \mathcal{P}_{\Omega}(X)$. Then by Lemma 2.6 we have

$$
d\left(\nu_{0}, \nu\right) \leq 4 \operatorname{diam} A \leq \frac{\varepsilon}{16} .
$$

From Lemma 2.5 we obtain

$$
\pi_{X} \Phi\left(n_{0}\right) \nu\left(K^{\frac{\varepsilon}{2}}\right) \geq \frac{\varepsilon}{2}
$$

for any $\nu \in \mathcal{P}_{\Omega}(X)$ supported on $\pi_{X}^{-1}(A)$. Notice that from definition of concentration we obtain $\pi_{X} \Phi(n) \mu(A) \geq \frac{\alpha}{2}$ for large enough $n \in \mathbb{N}$, which together with inequality (2.1) gives us $\pi_{X} \Phi(n) \mu \geq \frac{\alpha}{2} \pi_{X} \nu_{n}$ for large enough $n \in \mathbb{N}$. So we obtain

$$
\pi_{X} \Phi\left(n+n_{0}\right) \mu\left(K^{\frac{\varepsilon}{2}}\right) \geq \frac{\alpha}{2} \Phi\left(n_{0}\right) \nu_{n}\left(K^{\frac{\varepsilon}{2}}\right) \geq \frac{\alpha \varepsilon}{8}
$$


for large enough $n \in \mathbb{N}$. Since $K^{\frac{\varepsilon}{2}} \cap C_{\frac{\varepsilon}{2}}=\emptyset$ we have

$$
\begin{aligned}
\liminf _{n \rightarrow \infty} \pi_{X} \Phi(n) \mu\left(K^{\frac{\varepsilon}{2}} \cup C_{\frac{\varepsilon}{2}}\right) \geq \liminf _{n \rightarrow \infty} & \pi_{X} \Phi(n) \mu\left(K^{\frac{\varepsilon}{2}}\right) \\
& \quad+\liminf _{n \rightarrow \infty} \pi_{X} \Phi(n) \mu\left(C_{\frac{\varepsilon}{2}}\right) \geq \frac{\alpha \varepsilon}{8}+\delta>\delta .
\end{aligned}
$$

Since $K^{\frac{\varepsilon}{2}} \cup C_{\frac{\varepsilon}{2}}$ is $\frac{\varepsilon}{2}$-compact set this contradicts the definition of $\delta$.

Now we define recursively the numbers $n_{k}$, and measures $\nu_{k}, \mu_{k}$ as follows. Let $n_{0}=0, \mu_{0}=\nu_{0}=\mu$. Now if $n_{k-1}, \mu_{k-1}, \nu_{k-1}$ are given, we choose $n_{k}$ in accordance to the definition of concentration such that

$$
\pi_{X} \Phi\left(n_{k}\right) \mu_{k-1}(A) \geq \frac{\alpha}{2}
$$

and define

$$
\nu_{k}(B):=\frac{\Phi(n) \mu_{k-1}\left(B \cap \pi_{X}^{-1}(A)\right)}{\Phi(n) \mu_{k-1}\left(\pi_{X}^{-1}(A)\right)}
$$

for $B \in \mathcal{F} \times \mathcal{B}(X)$, and

$$
\mu_{k}(B):=\frac{1}{1-\frac{\alpha}{2}}\left(\Phi\left(n_{k}\right) \mu_{k-1}(B)-\frac{\alpha}{2} \nu_{k}(B)\right)
$$

for $B \in \mathcal{F} \times \mathcal{B}(X)$. By using this definitions we can observe that

$$
\begin{aligned}
\Phi\left(n_{1}+n_{2}+\ldots+n_{k}\right) \mu & =\frac{\alpha}{2} \Phi\left(n_{2}+\ldots+n_{k}\right) \nu_{1} \\
& +\frac{\alpha}{2}\left(1-\frac{\alpha}{2}\right) \Phi\left(n_{3}+\ldots+n_{k}\right) \nu_{2}+\ldots+\frac{\alpha}{2}\left(1-\frac{\alpha}{2}\right)^{k-1} \nu_{k}+\left(1-\frac{\alpha}{2}\right)^{k} \mu_{k}
\end{aligned}
$$

for any $k \in \mathbb{N}$. Now take $k \in \mathbb{N}$ such that $\left(1-\left(1-\frac{\alpha}{2}\right)^{k}\right)\left(1-\frac{\alpha}{2}\right) \geq 1-\varepsilon$. Then we have

$$
\begin{aligned}
\pi_{X} \Phi(n) \mu\left(C_{\frac{\varepsilon}{2}}^{\frac{\varepsilon}{2}}\right)= & \frac{\alpha}{2} \pi_{X} \Phi\left(n-n_{1}\right) \nu_{1}\left(C_{\frac{\varepsilon}{2}}^{\frac{\varepsilon}{2}}\right) \\
& +\ldots+\left(1-\frac{\alpha}{2} \Phi\left(n-n_{1}-\ldots-n_{k}\right) \nu_{k}\left(C_{\frac{\varepsilon}{2}}^{\frac{\varepsilon}{2}}\right)\right) \\
\geq & \left(1-\left(1-\frac{\alpha}{2}\right)^{k}\right)\left(1-\frac{\alpha}{2}\right) \geq 1-\varepsilon
\end{aligned}
$$

for $n \geq n_{1}+\ldots+n_{k}$. For smaller $n$ we can use Ulam's lemma to find a compact set $K \subseteq X$ such that

$$
\pi_{X} \Phi(n) \mu\left(K \cup C_{\frac{\varepsilon}{2}}^{\frac{\varepsilon}{2}}\right) \geq \pi_{X} \Phi(n) \mu(K) \geq 1-\varepsilon .
$$


We have shown that conditions of Lemma 2.3 are met, so we have tightness of the sequence $\{\Phi(n) \mu\}_{n \in \mathbb{N}}$, which by discussion in section 2.1 gives us the existence of an invariant measure.

Lemma 3.5. Let $\varphi$ be non-expansive. If for every $\varepsilon>0$ there exists $\alpha>0$ such that for every $\mu_{1}, \mu_{2} \in \mathcal{P}_{\Omega}(X)$ there exists $A \in \mathcal{B}_{b}(X)$ with $\operatorname{diam} A \leq \varepsilon$ and $n_{0} \in \mathbb{N}$ such that

$$
\pi_{X} \Phi\left(n_{0}\right) \mu_{i}(A)>\alpha
$$

for $i=1,2$, then

$$
\lim _{n \rightarrow \infty} d\left(\Phi(n) \mu_{1}, \Phi(n) \mu_{2}\right)=0
$$

for $\mu_{1}, \mu_{2} \in \mathcal{P}_{\Omega}(X)$

Proof. From Definition 2.4 it can be shown that if $\mu_{1}, \mu_{2}, \nu_{1}, \nu_{2} \in \mathcal{P}_{\Omega}(X)$ and $a, b \in \mathbb{R}$ are such that $a \mu_{1}+b \nu_{1}, a \mu_{2}+b \nu_{2} \in \mathcal{P}_{\Omega}(X)$ then

$$
d\left(a \mu_{1}+b \nu_{1}, a \mu_{2}+b \nu_{2}\right) \leq a d\left(\mu_{1}, \mu_{2}\right)+b d\left(\nu_{1}, \nu_{2}\right) .
$$

We will use this fact later on.

Let $\varepsilon>0$ and $\mu_{1}, \mu_{2} \in \mathcal{P}_{\Omega}(X)$. From assumption of the theorem take $\alpha>0, A \in \mathcal{B}_{b}(X)$ with $\operatorname{diam} A \leq \frac{\varepsilon}{4}$ and $n_{0} \in \mathbb{N}$ such that

$$
\pi_{X} \Phi\left(n_{0}\right) \mu_{i}(A)>\alpha
$$

for $i=1,2$. Let us define four sequences of measures $\left\{\mu_{i}^{k}\right\}_{k \in \mathbb{N}},\left\{\nu_{i}^{k}\right\}_{k \in \mathbb{N}}$ for $i=1,2$ such that $\mu_{i}^{0}=\nu_{i}^{0}=\mu_{i}$ for $i=1,2$ and having defined the sequences for $k-1$ we define

$$
\begin{aligned}
\nu_{i}^{k}(B) & :=\frac{\Phi\left(n_{0}\right) \mu_{i}^{k-1}\left(B \cap \pi_{X}^{-1}(A)\right)}{\pi_{X} \Phi\left(n_{0}\right) \mu_{i}^{k-1}(A)} \\
\mu_{i}^{k} & :=\frac{1}{1-\alpha} \Phi\left(n_{0}\right) \mu_{i}^{k-1}-\frac{\alpha}{1-\alpha} \nu_{i}^{k}
\end{aligned}
$$

for $i=1,2$. Then it is easy to see that

$$
\begin{aligned}
\Phi\left(k n_{0}\right) \mu_{i}=(1-\alpha)^{k} \mu_{i}^{k} & +\alpha \Phi\left((k-1) n_{0}\right) \nu_{i}^{1} \\
& +\alpha(1-\alpha) \Phi\left((k-2) n_{0}\right) \nu_{i}^{2}+\ldots+\alpha(1-\alpha)^{k-1} \nu_{i}^{k}
\end{aligned}
$$


for $i=1,2$. Since $\nu_{i}^{k} \in \mathcal{P}_{\Omega}^{\pi_{X}^{-1} A}(X)$ for $i=1,2$ and $k>1$ from Lemma 2.6 we obtain

$$
d\left(\nu_{1}^{k}, \nu_{2}^{k}\right) \leq 4 \operatorname{diam} A \leq \varepsilon
$$

for $k>1$. From this, the fact mentioned in the beginning of the proof and non-expansiveness of $\varphi$ and general fact that $d\left(\mu_{1}, \mu_{2}\right) \leq 2$ we get

$$
\begin{aligned}
d\left(\Phi\left(k n_{0}\right) \mu_{1}, \Phi\left(k n_{0}\right) \mu_{2}\right) \leq & (1-\alpha)^{k} d\left(\mu_{1}^{k}, \mu_{2}^{k}\right) \\
& +\alpha d\left(\Phi\left((k-1) n_{0}\right) \nu_{1}^{1}, \Phi\left((k-1) n_{0}\right) \nu_{2}^{1}\right) \\
& +\alpha(1-\alpha) d\left(\Phi\left((k-2) n_{0}\right) \nu_{1}^{2}, \Phi\left((k-2) n_{0}\right) \nu_{2}^{2}\right) \\
& +\ldots+\alpha(1-\alpha)^{k-1} d\left(\nu_{1}^{k}, \nu_{2}^{k}\right) \\
\leq & (1-\alpha)^{k} d\left(\mu_{1}^{k}, \mu_{2}^{k}\right)+\alpha d\left(\nu_{1}^{1}, \nu_{2}^{1}\right) \\
& +\ldots+\alpha(1-\alpha)^{k-1} d\left(\nu_{1}^{k}, \nu_{2}^{k}\right) \leq 2(1-\alpha)^{k}+\varepsilon .
\end{aligned}
$$

From non-expansiveness and the fact that $k$ and $\varepsilon$ were arbitrary we get the assertion.

THEOREM 3.6. If $\varphi$ is non-expansive and concentrating then $\varphi$ has the property of strong unique ergodicity.

Proof. Under the assumptions of the theorem we get from Lemma 3.4 the existence of an invariant measure $\nu \in \mathcal{I}_{\mathbf{P}}(\varphi)$. Since $\varphi$ is concentrating the assumptions of Lemma 3.5 are satisfied, using the conclusion of this lemma we obtain

$$
\lim _{n \rightarrow \infty} d(\Phi(n) \mu, \nu)=\lim _{n \rightarrow \infty} d(\Phi(n) \mu, \Phi(n) \nu)=0
$$

for all $\mu \in \mathcal{P}_{\Omega}(X)$. So $\varphi$ has the property of strong unique ergodicity.

\section{References}

[1] Arnold L., Random dynamical systems, Springer Monographs in Mathematics, Springer, Berlin, 1998.

[2] Crauel H., Random probability measures on Polish spaces, Series Stochastics Monographs, Vol. 11, Taylor \& Francis, London, 2002. 
[3] Crauel H., Flandoli F., Attractors for random dynamical systems, Probab. Theory Relat. Fields 100 (1994), 365-393.

[4] Lasota A., Yorke J.A., Lower bound technique for Markov operators and iterated function systems, Random Comput. Dynam. 2 (1994), 41-77.

[5] Szarek T., The stability of Markov operators on Polish spaces, Studia Math. 143 (2000), 145-152.

[6] Szarek T., Invariant measures for non-expansive Markov operators on Polish spaces, Dissertationes Math. 415 (2003), 62 pp.

[7] Valadier M., Young measures, in: Methods of Nonconvex Analysis (Varrenna 1989), Lecture Notes in Math. 1446, Springer, Berlin, 1990, pp. 152-188.

Institute of Mathematics

University of Silesia

BANKOWA 14

40-007 Katowice

POLAND

e-mail: pplonka@us.edu.pl 